\title{
Structural and Mechanical Characteristics of Metallic Glasses
}

\section{Chao Tang and Chee How Wong*}

School of Mechanical and Aerospace Engineering, Nanyang Technological University, 50 Nanyang Avenue, Singapore

Metallic Glasses (MGs) are potential candidate for a myriad of structural and engineering applications [1-3] because of their unique properties due in part of the absence of crystalline lattice which is found in conventional metals. However, the amorphous structure in MGs are still not very well understood and recent studies have highlighted the importance of understanding the atomic-level structure of MGs and its correlation with the mechanical behaviors [4].

To understand the structure of MGs, binary systems such as Ni-P and $\mathrm{Cu}-\mathrm{Zr}$ have been extensively studied [5-7]. Microscopically, the Short-Range Order (SRO) of MGs is characterized by the presence of similar solute-centered clusters $[5,8]$. The effective solute-to-solvent radius ratio plays an important role in determining the $\mathrm{SRO}$ in different systems [9-11]. The dominant SRO, or the structural order, could exhibit in the form of Kasper polyhedra with a large fraction of fivefold bonds [5]. However, any distortion to the fivefold bond will promote the formation of defective sites, which are energetically unstable $[4,5]$. Furthermore, the absence of direct solute-solute contact was confirmed by studying the partial Radial Distribution Functions (RDFs) [5]. At a larger scale, these similar clusters are efficiently packed and connected, forming the Medium-Range Order (MRO) in $3 \mathrm{D}$ space $[5,8,12]$.

Unlike in crystalline alloys where the lattice and crystal defects could be easily identified, in MGs, there are a variety of cluster types [5] resulting in heterogeneity in the structure. For example, some clusters such as Kasper polyhedra could be the dominant structure, while other clusters are relatively unstable and irregular. Egami et al. $[13,14]$ proposed the concept of atomic level stresses to explain the origins of these structural fluctuations. The size of each atom varies with its atomic level stress and leads to changes in the effective size ratio between the solute and the solvent atoms [13]. Since the effective size ratio determines the corresponding coordination number, therefore atoms with different stresses will possess different packing schemes. Furthermore, strain energy will be induced by the atomic level stresses [15], hence it is reasonable to conclude that atoms with lowest energy are stable and tend to form ordered clusters, whereas atoms with highest energy are likely to form structural defects.

The micro structural features of MGs are of fundamental importance for explaining their macroscopic properties, such as their strength, elasticity and deformation behavior. Although the mechanical performances of crystalline materials could be explained from the coexistence of lattice periodicity and crystal defects such as dislocations, the local topology varies from site to site in MGs, resulting in distinct mechanical behaviors at each site [4].

One interesting attribute of MGs is their ultrahigh strengthexceeding $1 \mathrm{GPa}$ in most cases [16,17]. The typical yield strain of MGs is about $2 \%$, which is far beyond that of crystalline alloys. While the strength of crystalline materials is determined by the sliding of dislocations, the strength of MG sis related to the strength of metallic bonds due to the absence of dislocations $[4,16]$. A well relaxed MG has a large fraction of unlike (solute-to-solvent) bonds which exhibit higher stability and bonding strength [4,5]. In other words, the solute atoms are likely to be surrounded by solvent atoms, leading to an improvement in strength [4].
At microscopic level, there are regions with low and high local stiffness in MGs [18]. This mechanical heterogeneity originates from the structural fluctuations in MGs, and has been confirmed by computer simulations [19-22]. Typically, Kasper polyhedra tend to percolate with each other, forming the structural backbone to sustain the entire matrix $[5,22]$. Consequently, regions with more Kasper polyhedra tend to form interpenetrating networks and exhibit higher resistance to external loads. Regions with less Kasper polyhedra are relatively unstable and are likely to undergo inelastic deformation. It is found that the shear resistance of MGs is enhanced with more rigid structural units. For instance, computer simulations on $\mathrm{Cu}-\mathrm{Zr} \mathrm{MG}$ confirmed that full icosahedra exhibits lower atomic strain energy and yields higher stiffness, which means that they are energetically more stable $[15,23]$. Furthermore, the icosahedra clusters tend to connect with one another, constituting the MRO in the system [12].

The yielding of MGs has been attributed to the percolation of Shear Transformation Zones (STZs) that undergo inelastic deformation and are characterized by the structural defects in MGs [24-26]. Typically, the STZs are dispersed in the matrix, which is supported by the structural backbone (networks of Kasper polyhedra). By applying a shear force onto the system, these STZs will experience inelastic deformations and gradually percolate with one another [27]. As long as the interconnection of these STZs does not exceed a critical length scale, they are reversible upon unloading. However, if the shear stress is sufficiently large, a variety of STZs will then connect with one another, giving rise to the destruction of the elastic backbone and irreversible plastic strain. At room temperature, shear deformation is concentrated in several localized shear bands, which is a catastrophic failure mode of MGs. Molecular Dynamics (MD) simulations have been applied to study the yielding and shear banding process of MGs $[28,29]$. For example, in $\mathrm{Cu}-\mathrm{Zr} \mathrm{MG}$, regions with more unstable polyhedra are likely to undergo inelastic deformations, while those with the more stable full icosahedra will only be elastically deformed. Furthermore, it is also reported that the full icosahedra in STZ should be transformed into other unstable clusters during the yielding process.

In summary, the atomic structure of MGs has a broad distribution of networks that gives rise to their unique mechanical properties. Although the mechanical behaviors of MGs have been intensively analyzed through experiments, understanding of the underlying mechanisms of their mechanical performances is still inadequate.

*Corresponding author: Chee How Wong, School of Mechanical and Aerospace Engineering, Nanyang Technological University, 50 Nanyang Avenue, Singapore 639798, Singapore, Tel: +65 6790 5913; Fax: +65 6792 4062; E-mail: chwong@ntu.edu.sg

Received October 22 2013; Accepted October 22, 2013; Published October 25 , 2013

Citation: Tang C, Wong CH (2013) Structural and Mechanical Characteristics of Metallic Glasses. J Appl Mech Eng 2: e120. doi:10.4172/2168-9873.1000e120

Copyright: () 2013 Tang C, et al. This is an open-access article distributed under the terms of the Creative Commons Attribution License, which permits unrestricted use, distribution, and reproduction in any medium, provided the original author and source are credited. 
Citation: Tang C, Wong CH (2013) Structural and Mechanical Characteristics of Metallic Glasses. J Appl Mech Eng 2: e120. doi:10.4172/21689873.1000e120

Henceforth, the atomic structure and structure-property relationship of MGs need further investigations to unravel and improve our understanding of the glassy state.

\section{References}

1. Ashby MF, Greer AL (2006) Metallic glasses as structural materials. Scripta Mater 54: 321-326.

2. Greer AL, Ma E (2007) Bulk metallic glasses: At the cutting edge of metals research. MRS Bulletin 32: 611-619.

3. Wang WH, Dong C, Shek C (2004) Bulk metallic glasses. Mater Sci Eng: R: Reports 44: 45-89.

4. Cheng YQ, Ma E (2011) Atomic-level structure and structure-property relationship in metallic glasses. Prog Mater Sci 56: 379-473.

5. Sheng HW, Luo WK, Alamgir FM, Bai JM, Ma E (2006) Atomic packing and short-to-medium-range order in metallic glasses. Nature 439: 419-425.

6. Tang MB, Zhao DQ, Pan MX, Wang WH (2004) Binary Cu-Zr bulk metallic glasses. Chinese Phys Lett 21: 901

7. Wang D, Li Y, Sun BB, Sui ML, Lu K, Ma E (2004) Bulk metallic glass formation in the binary $\mathrm{Cu}-\mathrm{Zr}$ system. Appl Phys Lett 84: 4029-4031.

8. Miracle DB (2004) A structural model for metallic glasses. Nature materials 3 : 697-702.

9. Miracle DB (2006) The efficient cluster packing model-An atomic structura model for metallic glasses. Acta Mater 54: 4317-4336.

10. Miracle DB (2004) Efficient local packing in metallic glasses. J non-cryst solids 342: 89-96.

11. Cheng YQ, Ma E, Sheng HW (2009) Atomic level structure in multicomponent bulk metallic glass. Phys Rev lett 102: 245501.

12. Wang $\mathrm{CC}$, Wong $\mathrm{CH}$ (2012) Interpenetrating networks in $\mathrm{Zr}-\mathrm{Cu}-\mathrm{Al}$ and $\mathrm{Zr}-\mathrm{Cu}$ metallic glasses. Intermetallics 22: 13-16.

13. Egami T (2011) Atomic level stresses. Prog Mater Sci 56: 637-653.

14. Vitek V, Egami T (1987) Atomic level stresses in solids and liquids. physica status solidi 144 : 145-156.
15. Cheng YQ, Ding J, Ma E (2012) Local Topology vs. Atomic-Level Stresses as a Measure of Disorder: Correlating Structural Indicators for Metallic Glasses. Materials Research Letters 1: 3-12.

16. Suryanarayana C, Inoue A (2011) Bulk metallic glasses: Taylor \& Francis, US.

17. Wang WH (2012) The elastic properties, elastic models and elastic perspectives of metallic glasses. Prog Mater Sci 57: 487-656.

18. Liu YH, Wang D, Nakajima K, Zhang W, Hirata A, et al. (2011) Characterization of nanoscale mechanical heterogeneity in a metallic glass by dynamic force microscopy. Phys rev lett 106: 125504.

19. Albano F, Lacevic N, Falk ML, Glotzer SC (2004) Relating metallic glass mechanical properties to liquid structure. Mater Sci Eng: A 375: 671-674.

20. Albano F, Falk ML (2005) Shear softening and structure in a simulated threedimensional binary glass. J chem phys 122: 154508.

21. Shi Y, Falk ML (2005) Structural transformation and localization during simulated nanoindentation of a noncrystalline metal film. Appl Phys Lett 86.

22. Shi Y, Falk ML (2006) Does metallic glass has a backbone? The role of percolating short range order in strength and failure. Scriptamaterialia 54: 381 386.

23. Cheng Y, Cao A, Ma E (2009) Correlation between the elastic modulus and the intrinsic plastic behavior of metallic glasses: The roles of atomic configuration and alloy composition. ActaMaterialia 57: 3253-3267.

24. Argon A (1979) Plastic deformation in metallic glasses. Acta Metall 27: 47-58.

25. Langer JS (2006) Shear-transformation-zone theory of deformation in metallic glasses. Scriptamaterialia 54: 375-379.

26. Pan D, Inoue A, Sakurai T, Chen M (2008) Experimental characterization of shear transformation zones for plastic flow of bulk metallic glasses. Proceedings of the National Academy of Sciences 105:14769-14772.

27. Harmon JS, Demetriou MD, Johnson WL, Samwer K (2007) Anelastic to plastic transition in metallic glass-forming liquids. Phys rev lett 99: 135502.

28. Shi Y, Falk ML (2007) Stress-induced structural transformation and shea banding during simulated nanoindentation of a metallic glass. Actamaterialia 55: 4317-4324.

29. Cao AJ, Cheng YQ, Ma E (2009) Structural processes that initiate shea localization in metallic glass. Actamaterialia 57: 5146-5155. 